\title{
Analysis of Recent Trend and Applications in Block Chain Technology
}

\author{
Vivekanadam B \\ Professor, \\ Lincoln University, \\ Malaysia. \\ vivekanandam@lincoln.edu.my
}

\begin{abstract}
Blockchain is a digital ledger in which each record known as blocks and that are combined in a single list known as a chain. It is regarded as Bitcoin's backbone technology. It is also regarded as cohesive collections of digital wallets. Blockchains are primarily used by cryptocurrencies such as Bitcoin and other applications to record these transactions. A blockchain is commonly referred to as a collection of distributed databases that consists of all public transactions, records and digital events then that information is shared among the participants. Every transaction is verified and it cannot be removed. The main features of this technology are reliable, efficient operation, fault tolerance and scalability. Some of the applications are manufacturing, government and finance when the three properties met together (i.e., Efficiency, Scalability and Security). By using several computers, each transaction that is applied to a blockchain is validated. A peer-to-peer network is developed by these systems that are used to validate these forms of blockchain transactions. They work together to ensure that any transaction is legitimate until it is added to the blockchain, and invalid blocks cannot be added to the chain by these systems. When a new block is added, it can be connected to a previous block using a cryptographic hash and the chain cannot be broken and each block is recorded permanently. Blockchain can be used for an exchanging the transaction securely without an intermediate. It enables customer relationship and agile chain values and thereby integrating with IoT and Cloud technology. The functionality of distributed ledger is combined with blockchain security to solve the financial and non-financial industry problems. This paper proposes the blockchain technology with devices and creates a common platform and secure data communication.
\end{abstract}

Keywords:- Bitcoins, Blockchain Technology, Security, Transactions, Cryptography, Cryptocurrency

\section{Introduction}

Blockchain Technology is developed to support the cryptocurrency blockchain to make use of the transaction without an intermediator. The first blockchain-based cryptography is Bitcoin. In this bitcoin, all the information represents the electronic cash that is attached to the digital address and it allows the users to digitally sign and it transfers the rights to other persons. It stored, maintained and managed by a group of participants [30]. Bitcoin is considered as peer-to-peer ledger technology that used for agreements, sales, contracts and recording the transactions. In Bitcoin, there is no guarantee that the node received the transaction as per the order in which it is generated. So, it is important to build a mechanism for the Bitcoin network for agreeing to the transaction order. Then the problem is solved by a popular mechanism is known as Blockchain. A ledger is defined as a collection of transactions.

The main advantage of blockchain is it is impossible to initiate an attack in the blockchain network because it has to compromise $51 \%$ of its systems to the target network [29]. It also serves as an immutable ledger and all the transactions are decentralized. Blockchain characteristics are audibility, persistency, anonymity and decentralization. Thereby efficiency is increased and the cost is saved. Blockchain is applied in many financial services like online payments and digital assets and also used in the Internet of Things (IoT), Security and Public services [4]. Blockchain is immutable so that it can be applied in different fields. It is reliable and distributed to attract a large number of customers for business-related purposes. Figure 1 shows the features of the blockchain. It avoids the single point of failure. For making transactions in the blockchain, the users can also use public and private keys [24]. Within the blockchain network, an individual system is known as a node. It is classified into three modes such as publishing node, a full node and lightweight node. In publishing node, it publishes new blocks [6]. In the full node, it stores the whole blockchain and it checks for the valid transactions. In a lightweight node, it does not store any blockchain copies and the transactions are transferred to the full nodes. 
Journal of ISMAC (2020)

Vol.02/ No.04

Pages: 200-206

http://irojournals.com/iroismac/

DOI: https://doi.org/10.36548/jismac.2020.4.003

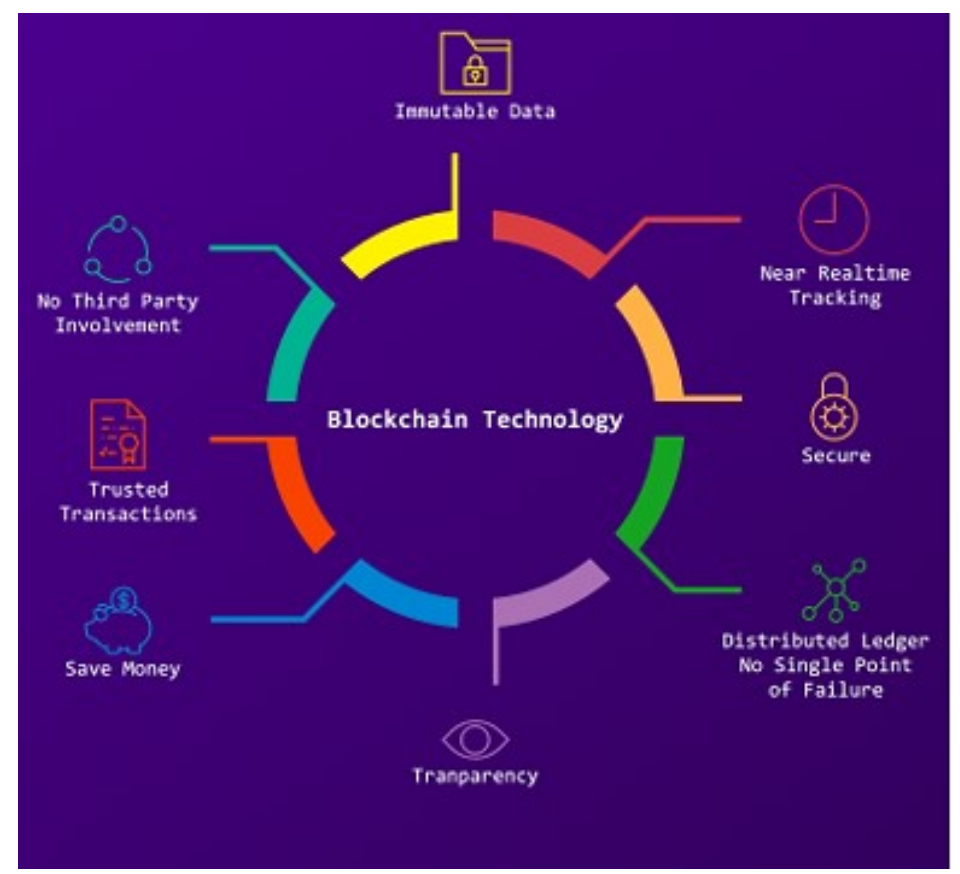

Fig.1 Features of Blockchain Technology

Blockchain is mainly composed of a sequence of blocks and it is connected linked to the previous block cryptographically. It consists of a block header and block body. It consists of a nonce, parent block hash, N-bits, Merkle tree root hash and block version are included in the block header. The block body composed of transactions and the counter of transactions [8]. To validate the transaction authentication, blockchain uses asymmetric cryptography. Three forms of blockchain are categorized such as consortium blockchain, private blockchain and private blockchain. The private blockchain is only for the particular nodes that arrives from a particular organization and then passes to the subsequent process [25]. It is also known as a centralized network and it is controlled by a single organization. In consortium blockchain process, a group of pre-selected nodes only could join in the consensus process and it is partially decentralized.

Based on the comparison of the types of blockchain it is further explained as the property of blockchain such as read permission, efficiency, immutability, consensus determination and process then centralized [19]. The consensus is the main part in public blockchain and it has a set of nodes for validation and private chain gives the final consensus. The transactions are available to the public inside a transparent blockchain. It is not possible to interrupt transactions when records of more users are kept. But in the private blockchain, it is easy for only when it is used for a smaller number of participants. It consumes more time during transactions on the public blockchain. Due to the result of the transactions, it produces high latency and limited throughput [10].

A public blockchain is a decentralized and fully centralized in a private blockchain, with the consortium being partially centralized. Figure 2 explains the working of blockchain. The public blockchain allows to visible all the records. [20]. In the blockchain, it has several challenges and it decreases the limit of blockchain due to privacy leakage, scalability and selfish mining. A million of transactions is happening every day and the blockchain becomes a bottleneck due to small block size and it has to store all the transactions and making corrections [21]. To address the problem of scalability, redesigning blockchain and storage optimization of blockchain is used. In privacy leakage, it does not have any transactional privacy because all the transactions and the public key is visible to everyone. In the smart city, the data is transferred securely using blockchain technology without compromising the security and privacy in the network. Considering the smart cities, it is open to threats and the possibility of threats are classified as Threats on Accountability, Threats on Confidentiality,

Threats on Availability, Threats on Integrity and Threats on Authenticity. Then the required security framework was built on the requirements of smart cities. It mainly consists of all layers and includes interface and database layer [15-18]. In Physical Layer, it consists of sensors and actuators that used for collecting the data and transfers to the next layer. In Communication Layer, it uses different networks and protocols for communications such as $\mathrm{Wi}-\mathrm{Fi}, 3 \mathrm{G}$ and $4 \mathrm{G}$, Bluetooth for exchanging the information. All the blockchain protocols must be collaborated with this communication layer for providing privacy and security. In the Database Layer, it consists 
Journal of ISMAC (2020)

Vol.02/ No.04

Pages: 200-206

http://irojournals.com/iroismac/

DOI: https://doi.org/10.36548/jismac.2020.4.003

of Distributed Ledger as a decentralized database for storing the records. Each record holds the unique cryptographic signature and timestamp. It checks for the authenticated user [12]. There are two types of ledger such as private (Permissioned) and public ledger (Permissionless). For ensuring the performance, security and scalability it is better to use private ledgers. In Interface Layer, it consists of several applications to make effective decisions.

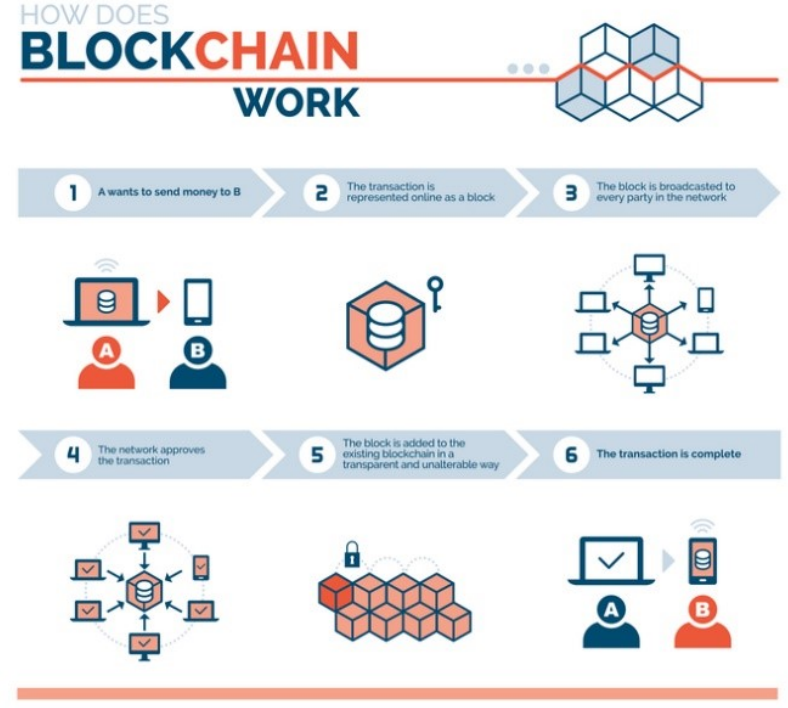

Fig. 2 Working of Blockchain

The two main important features of blockchain technology are anonymity and distributed consensus. Smart Contracts allows executing the contract automatically with the help of computer programs [22]. It is based on the contractual agreements between the parties and the payment has been made as per the agreement. Another concept that is closely related to the smart contract is Smart Property used for controlling the property ownership using blockchain technology. The property can be anything (i.e.,) household property or company shares. It is possible to use blockchain in both financial and non-financial terms. Financial transactions deal with institutions and banks for security purposes and non-financial transactions related to records, legal documents and payments. It is carried out by storing the digital asset fingerprint instead of the digital asset with this method privacy and anonymity can be preserved.

To improve the efficiency of blockchain, it uses anonymous and mixing. It is categorized into two approaches of blockchain they are permissioned and permissionless [11]. In permissioned blockchain, it limits the user and permits finer-grained controls. In a permissionless blockchain, it allows everyone to read and write to the blockchain without any authorization. Blockchain is spanned from many applications both financial and nonfinancial and there are some risks of adoptions. Some of the risks of adoptions are behaviour change, bootstrapping, government regulations, quantum computing, fraudulent activities and scaling. Behaviour change represents constant change. In this trusted third parties uses blockchain for safe and secured transactions. Bootstrapping moves the existing documents and contracts to the new framework. Scaling represents the blockchain services by validating the transactions when the number of users increases [14]. In government regulations, it comprises of agencies like SEC and FTC by monitoring and regulating the industry compliance. Fraudulent activities deal with the misusing the blockchain transactions like money trafficking by enforcing the law and regulations for monitoring the individuals. Quantum computing is the basis of blockchain technology and it needs power computing. It uses cryptographic keys using sheer brute force approaches [13]. The future of blockchain is respect to four directions such as big data analytics, stopping the tendency to centralization and blockchain testing. In the blockchain testing, more than 700 cryptocurrencies are specified [8]. It is further classified into two phases they are testing and standardization phases. In the testing phase, it has to be tested with various parameters and in the standardization phase all the criteria are made and it is agreed if it is valid. Blockchain is integrated with big data for storing and managing data. Then it should be secured and data integrity 
is preserved. For analyzing and predicting the transaction data with the partner's blockchain utilizes big data analytics [24]. There are many applications regarding blockchain one such application is a smart contract that executes the contract and it is computerized based transaction-based protocol. This technology combines the proven concepts and existing problem then merges to give a single solution.

\section{Literature Survey}

The research paper named Innovations of Blockchain Technology was proposed by Tareq Ahram et al. The blockchain would entitle added chains of nimble value with innovations of brisk outcomes in the neighbourhood of customer association and meteoric amalgamation with the technology of cloud and Internet of Things. Additionally, it furnishes commerce of minimal expenditure unescorted from minor parties by the mediation who ought to put-on merit but with a certitude monitored commitment [9]. It smoothens smart covenants, arrangement and concords with immanent, vigorous features of virtual security. This proposed system is an attempt to snap the premise for attending and illustrating the ply in variant manufacturing applications of Blockchain technology. In an industrial application using Blockchain of IBM inventiveness the health chain is standardized and expanded on the underpinning of Blockchain. The abstraction is interchangeable to all-inclusive of fabrication where safety, extensibility and productivity must comply such as finance, government and manufacturing. The objectives and constituents were selected to set the seal on a sturdy architecture based on breaches of cyber-security for the precaution, segregation and amendatory. The manifesto is accustomed to disentangle the superintendence of who can indicate to cloud technologies and scrutinize that entreaty for weaknesses, submerged dominance of security facing ministrations of big data and guidance of information. Communal groundwork is flattering more make headway through detectors, computing splinters, finer power founts and actuators.

The Blockchain technology gapes to executive mastermind to assist controls and its progression superintend. The transformations of crucial potency industry are more likely to crop up as distributed ledger fullyfledged. In the first instance favoured by Bitcoin and the Blockchain is immeasurably more than the understructure meant for cryptocurrency. Blockchain also known as groupware technology will needless to say aptly onto the salaried engineering of software authorize minutest care streamer. Ample has been attained by inaugurating the mobile applications and impersonal routines software so far in the face of the encryption conventionally and backdrops of countersign can be auditioned by Health Insurance Portability and Accountability Act for an engulfed system and rupture stock still occur also Protected Health Information would be accommodated [23]. The latest wheel of life the information for protected health on the contributor networks of the wellness program.

An application which is cloud-based known as the Health Chain and the investigators has exemplifies the potentiality to appeal propositions of white-collar engineering incorporated with an evolving strategy into continual development and administration with the unification of distributed computing, technologies of Blockchain and cybersecurity [7]. They perceive Health Chain to be one of the best among many exemplars that evidence the reframing proficiency of Block bond. Blockchain is used in cryptocurrency Bitcoin. Blockchain is a simplified payment verification system. It is used in financial and non-financial industries in the field like Manufacture industry, supply chain and health care. This study plays a pivotal role in performing digitization of industries. The cybersecurity provides security and privacy control to particular data and also play a major role in data management includes data masking, discovery and audit. And it may reuse essential processes and trends from end to end to drive the performance of the information system and the life cycle.

The analogue to the digital computer by conversion process serves the professional duty in this blockchain. These processes carried out by software usages. Sensors, computer chips, actuators and power sources are now becoming more advanced in public infrastructure [26]. All the digitalization of transport, air quality, water purity, structures, products, etc. are transformed through software, hardware and network systems. Professionals may now safeguard public safety and protection and prioritise health and welfare. Information technology in almost every sector is a vital breakthrough. In a leadership position, such innovations effectively play a significant role.Blockchain Technology and its architecture were suggested by Zibin Zheng et al. This paper offers a thorough recapitulation on the technology of blockchain. They dispense an outline of blockchain framework initially and collate few prototypical concords algorithms in dissimilar block bonds can be used. Moreover, the non-theoretical provocations and latest cultivates are quickly listed [2]. For blockchain, they also provide feasible future directions. This technology is allocated to keep away from the neglect circumstances of solitary perspective.

The commitment would be implemented by faceworkers automatically only once approval has been inclined on the blockchain for the remote contracts. Although the blockchain technology has the greatest dormant for the construction of future Internet networks, many technological challenges are facing it. It instantiates a 
sample of the blockchain. A block has a single parent block accompanied by premature block hemp repressed in the block caption. In an ethereum of blockchain, the matured blocks hashes would also be stockpiled which is unworthy [28]. With the communal blockchain, every single evidence is discernable through to be publicized, all and sundry would play a part in the process of concurrence. Contrastingly narrowly a variety of initially-selected junctions will engage in the conglomerate blockchain for consensus task. As for the non-public blockchain, the nodes that are special that always arrive from a particular dovetail will be permitted to join the consensus action. A blockchain which, because it is completely regulated by one organisation, is considered to be privately chosen as a central network. There is no central junction in the blockchain that ensures the ledgers on scatter nodes are all identical. To ensure that records are consistent with different nodes, some protocols are required.

A nonce was restrained by the block header and miners would habitually change the nonce to get different dope values. Valid blocks may be generated simultaneously in the decentralised network when different nodes almost at the same time find suitable nonce. To create and certify blocks, stakeholders elect their delegates. The block could be easily authenticated with slightly lower nodes to bear out the block, resulting in fast negotiation confirmation. In the meantime, delegates may change the network guidelines, such as block footage and block spans [3]. In a round, a new block is decided. A proposer in this round will be chosen to circulate an unconfirmed block. The individuality of the node is expected to be known to the whole network, so it could be used in commercial rather than the public mode. On the blockchain, every one node has to reserve altogether give away to collaborate them since they have to inspect if the wellspring is amassed or not of the ongoing transaction. For the time being as the block amplitude is extremely negligible following miners lodge these specified transactions escorted by towering allowance for the transaction, countless minuscule transactions would be retarded [27]. This proposed protocol cleaves duration as periods. Per capita, period hewers have to be hashed to engender a unique key structure.

The node embellishes the superior who is blameworthy for spawning the micro blocks at one time the key complex is also produced. The testing phases of Blockchain can be further detached likely as standardization and examining phase. In the introductory phase of the blockchain, every yardstick has to be assembled and sympathized [5]. Whenever a blockchain is inherent, it should be evaluated upon the acknowledged basis geared toward validation if the concerned blockchain operates at first-rate as makers profess. Moreover, we have sorted a few oppositions and drawbacks that may retard the phenomenon of blockchain and encapsulated sundry approaches which are subsisting for resolving these complications. Some of the workable succeeding supervisions are also offered.Nadir Abdelrahman Ahmed Farah conducted a study on Blockchain technology is a structure that stores transactional records to increase the decentralization transparency, equality and responsibility of the internet. The main advantage is it was stored as a block very difficult to hack. Blockchain provides higher security when compared with central database [31]. It prevents harm from attacks by using specialized technologies in Bitcoin mines.

The blockchain is made up of key data, the previous block hash, the new bock time stamp hash, and other data. Figure 3 shows the standard vs blockchain transactions. Blockchain classified into four types of permissionless. Permissioned, public and private. Under a consensus mechanism, the public or permissionless blockchain is used for proof of work and proof of interest. Another type of permission blockchain used for business. In a private blockchain, permission is given under the central organization. Then comparing standard vs blockchain-based transaction it shows the difference between certain models such as paradigm, architecture, database, security, cost prize and access. The financial contracts provide security to the counterparties and it helps to record all the contracts and it enhances to understand the updates and events in the market. About the asset tracking, it helps in addressing the stolen goods in international supply.

Table 1 Standard transactions Vs Block chain transactions

\begin{tabular}{|c|c|c|}
\hline Model & Standard & Blockchain \\
\hline Paradigm & Trusted third party & Trusties system \\
\hline Architecture & Centralized & Peer to peer \\
\hline Database & Single copy & Multiple Copies \\
\hline Security & Controlled access & Cryptography \\
\hline Price & Intermediate & Consensus \\
\hline Access & Private & Public \\
\hline
\end{tabular}

The digital identity in blockchain used to identify and track the ownership and goods and identity of people also it can able to track [1]. These different blockchain systems against various parameters like 
Journal of ISMAC (2020)

Vol.02/ No.04

Pages: 200-206

http://irojournals.com/iroismac/

DOI: https://doi.org/10.36548/jismac.2020.4.003

performance cost efficiency and flexibility. Bitcoin network consists of a group of globally distributed computers all under open-source software. Blockchain used for many applicators. The available use case for blockchain is an asset tracking tool for enhancing proof of ownership for a particular asset. The opportunities include it affected the transforming of current internet from information sharing to the value of the exchange. This paper discussed the blockchain technology and it's important. The challenges in the blockchain are for non-fiat currency. The blockchain challenges include blockchain capacity, locked-in funds and lack of solid anonymity. This technology will improve the status of industries and it used to avoid hacking and forgery activities.

\section{Conclusion}

Blockchain technology is regarded as a new tool for organizations with many potential applications for secure transactions without an intermediator. Blockchain has been used for financial and non-financial industries for various purposes like manufacturing, healthcare industries and supply chain. Blockchain permits agreements and contracts inherently with security features and enables customer relationship directly with IoT and cloud technologies. It can able to solve the industrial problems and trusted third party is present for marketing services. It introduces a new digital business model and health initiatives in healthcare. It creates a common platform for devices to communicate in a distributed environment securely. Blockchain possesses low trading cost, exchanging of information securely and enables high agile value. In future, it works on the development of scalability and interoperability features in the blockchain technology for secured transactions.

\section{References}

1. Crosby, M., Pattanayak, P., Verma, S., \& Kalyanaraman, V. (2016). Blockchain technology: Beyond bitcoin. Applied Innovation, 2(6-10), 71.

2. Zheng, Z., Xie, S., Dai, H., Chen, X., \& Wang, H. (2017, June). An overview of blockchain technology: Architecture, consensus, and future trends. In 2017 IEEE international congress on big data (BigData congress) (pp. 557-564). IEEE.

3. Yli-Huumo, J., Ko, D., Choi, S., Park, S., \& Smolander, K. (2016). Where is current research on blockchain technology? - a systematic review. PloS one, 11(10), e0163477.

4. Pilkington, M. (2016). Blockchain technology: principles and applications. In Research handbook on digital transformations. Edward Elgar Publishing.

5. Mettler, M. (2016, September). Blockchain technology in healthcare: The revolution starts here. In 2016 IEEE 18th international conference on e-health networking, applications and services (Healthcom) (pp. 1-3). IEEE.

6. Biswas, K., \& Muthukkumarasamy, V. (2016, December). Securing smart cities using blockchain technology. In 2016 IEEE 18th international conference on high performance computing and communications; IEEE 14th international conference on smart city; IEEE 2nd international conference on data science and systems (HPCC/SmartCity/DSS) (pp. 1392-1393). IEEE.

7. Yaga, D., Mell, P., Roby, N., \& Scarfone, K. (2019). Blockchain technology overview. arXiv preprint arXiv:1906.11078.

8. Saberi, S., Kouhizadeh, M., Sarkis, J., \& Shen, L. (2019). Blockchain technology and its relationships to sustainable supply chain management. International Journal of Production Research, 57(7), 2117-2135.

9. Ahram, T., Sargolzaei, A., Sargolzaei, S., Daniels, J., \& Amaba, B. (2017, June). Blockchain technology innovations. In 2017 IEEE technology \& engineering management conference (TEMSCON) (pp. 137141). IEEE.

10. Beck, R., Avital, M., Rossi, M., \& Thatcher, J. B. (2017). Blockchain technology in business and information systems research.

11. Sun, J., Yan, J., \& Zhang, K. Z. (2016). Blockchain-based sharing services: What blockchain technology can contribute to smart cities. Financial Innovation, 2(1), 1-9.

12. Treleaven, P., Brown, R. G., \& Yang, D. (2017). Blockchain technology in finance. Computer, 50(9), 14-17.

13. Sikorski, J. J., Haughton, J., \& Kraft, M. (2017). Blockchain technology in the chemical industry: Machine-to-machine electricity market. Applied energy, 195, 234-246.

14. Andoni, M., Robu, V., Flynn, D., Abram, S., Geach, D., Jenkins, D., ... \& Peacock, A. (2019). Blockchain technology in the energy sector: A systematic review of challenges and opportunities. Renewable and Sustainable Energy Reviews, 100, 143-174. 
Journal of ISMAC (2020)

Vol.02/ No.04

Pages: 200-206

http://irojournals.com/iroismac/

DOI: https://doi.org/10.36548/jismac.2020.4.003

15. Meng, W., Tischhauser, E. W., Wang, Q., Wang, Y., \& Han, J. (2018). When intrusion detection meets blockchain technology: a review. Ieee Access, 6, 10179-10188.

16. Chen, G., Xu, B., Lu, M., \& Chen, N. S. (2018). Exploring blockchain technology and its potential applications for education. Smart Learning Environments, 5(1), 1.

17. Min, H. (2019). Blockchain technology for enhancing supply chain resilience. Business Horizons, 62(1), $35-45$.

18. Angraal, S., Krumholz, H. M., \& Schulz, W. L. (2017). Blockchain technology: applications in health care. Circulation: Cardiovascular quality and outcomes, 10(9), e003800.

19. Tian, F. (2016, June). An agri-food supply chain traceability system for China based on RFID \& blockchain technology. In 2016 13th international conference on service systems and service management (ICSSSM) (pp. 1-6). IEEE.

20. Gordon, W. J., \& Catalini, C. (2018). Blockchain technology for healthcare: facilitating the transition to patient-driven interoperability. Computational and structural biotechnology journal, 16, 224-230.

21. Wright, A., \& De Filippi, P. (2015). Decentralized blockchain technology and the rise of lex cryptographia. Available at SSRN 2580664.

22. Hawlitschek, F., Notheisen, B., \& Teubner, T. (2018). The limits of trust-free systems: A literature review on blockchain technology and trust in the sharing economy. Electronic commerce research and applications, 29, 50-63.

23. Turk, Ž., \& Klinc, R. (2017). Potentials of blockchain technology for construction management. Procedia engineering, 196, 638-645.

24. Cocco, L., Pinna, A., \& Marchesi, M. (2017). Banking on blockchain: Costs savings thanks to the blockchain technology. Future internet, 9(3), 25.

25. Zhang, P., Schmidt, D. C., White, J., \& Lenz, G. (2018). Blockchain technology use cases in healthcare. In Advances in computers (Vol. 111, pp. 1-41). Elsevier.

26. Singh, M., \& Kim, S. (2018). Branch based blockchain technology in intelligent vehicle. Computer Networks, 145, 219-231.

27. Joshi, A. P., Han, M., \& Wang, Y. (2018). A survey on security and privacy issues of blockchain technology. Mathematical foundations of computing, 1(2), 121.

28. Pan, X., Pan, X., Song, M., Ai, B., \& Ming, Y. (2020). Blockchain technology and enterprise operational capabilities: An empirical test. International Journal of Information Management, 52, 101946.

29. Hou, H. (2017, July). The application of blockchain technology in E-government in China. In 201726 th International Conference on Computer Communication and Networks (ICCCN) (pp. 1-4). IEEE.

30. Miraz, M. H., \& Ali, M. (2018). Applications of blockchain technology beyond cryptocurrency. arXiv preprint arXiv: 1801.03528 .

31. Farah, N. A. A. (2018). Blockchain Technology: Classification, Opportunities, and Challenges. International Research Journal of Engineering and Technology, 5(5), 3423-3426. 